\title{
cccreative
}

ISSN 2590-9770

The Art of Discrete and Applied Mathematics 2 (2019) \#P2.06

https://doi.org/10.26493/2590-9770.1333.152

(Also available at http://adam-journal.eu)

\section{Regular antilattices}

\author{
Karin Cvetko-Vah * \\ Department of Mathematics, Faculty of Mathematics and Physics, \\ University of Ljubljana, Jadranska 21, SI-1000 Ljubljana, Slovenia
}

\section{Michael Kinyon ${ }^{\dagger}$}

Department of Mathematics, University of Denver, Denver, CO 80208, USA

\section{Jonathan Leech}

Department of Mathematics, Westmont College, Santa Barbara, CA 93108, USA

\section{Tomaž Pisanski ${ }^{\ddagger}$}

Faculty of Mathematics, Natural Sciences and Information Technologies,

University of Primorska, Glagoljaška 8, SI-6000 Koper, Slovenia, and

Andrej Marušič Institute, University of Primorska,

Muzejski trg 2, SI-6000 Koper, Slovenia, and

Faculty of Mathematics and Physics, University of Ljubljana,

Jadranska 21, SI-1000 Ljubljana, Slovenia, and

Institute of Mathematics, Physics and Mechanics (IMFM),

Jadranska 19, SI-1000 Ljubljana, Slovenia

Received 18 November 2019, accepted 21 December 2019, published online 30 December 2019

\begin{abstract}
Antilattices $(S ; \vee, \wedge)$ for which the Green's equivalences $\mathcal{L}_{(\vee)}, \mathcal{R}_{(\vee)}, \mathcal{L}_{(\wedge)}$ and $\mathcal{R}_{(\wedge)}$ are all congruences of the entire antilattice are studied and enumerated.

Keywords: Noncommutative lattice, antilattice, Green's equivalences, lattice of subvarieties, enumeration, partition, composition.

Math. Subj. Class.: 06B75, 05A15, 05A17, 03G10, 11 P99

\footnotetext{
* Corresponding author.

${ }^{\dagger}$ Michael Kinyon was supported by the Simons Foundation Collaboration Grant 359872.

¥Work of Tomaž Pisanski is supported in part by the ARRS grants P1-0294, J1-7051, N1-0032, and J1-9187.

E-mail addresses: karin.cvetko@fmf.uni-lj.si (Karin Cvetko-Vah), michael.kinyon@du.edu (Michael
} Kinyon), leech@westmont.edu (Jonathan Leech), pisanski@upr.si (Tomaž Pisanski)
\end{abstract}

()( This work is licensed under https://creativecommons.org/licenses/by/4.0/ 


\section{Introduction}

In the study of noncommutative lattices, lattices still play an important role. They are the commutative cases of the algebras being considered and indeed play an important role in the general theory of that larger class of algebras. (As with rings, "noncommutative" is understood inclusively to mean not necessarily commutative). But also, typically, a second subclass of algebras exists that plays counterpoint to the subclass of lattices. It has become common to refer to their members as "antilattices." Typically they resist any kind of nontrivial commutative behavior. That is, an instance of $x y=y x$ for a relevant binary operation can occur only when $x=y$. Antilattices, however, are not without their special charm. Indeed, they have been studied in connection with magic squares and finite planes. (See [8].)

In this paper we study the class of regular antilattices for which the Green's equivalences are congruences. Precise definitions occur in Section 2 where basic concepts such as bands, quasilattices and the condition of regularity are described, along with some relevant preliminary results.

Regular antilattices themselves are the focus of Section 3. The main results are a very precise decomposition given in Theorem 3.3 and its several consequences. A closer look at the lattice of subvarieties (see Figure 1) occurs in the final fourth section.

The reader seeking further information on bands is referred to the presentations given in Clifford and Preston [4], in Grillet [5] and in Howie [6]. For further background on skew lattices and quasilattices, see [7] and [9]. The basic facts of universal algebras, and in particular varieties, may be found in the second chapter of [2].

\section{Preliminary concepts and results}

A band is a semigroup $(S ; \cdot)$ for which all elements are idempotent, that is, $x x=x$ holds. A band is rectangular if it satisfies the identity $x y z=x z$, or equivalently, $x y x=x$. (As often occurs, if just a single binary operation is involved, its appearance is suppressed in equations.) A semilattice is a commutative band $(x y=y x)$. Clearly, rectangular semilattices form the class of trivial 1-point bands. Indeed both classes are structural opposites that play important roles in the general structure of bands. To see how and to set the stage for further preliminaries requires the use of Green's relations, defined first for bands.

$$
\begin{array}{clll}
\mathcal{D}: & x \mathcal{D} y & \text { iff } & \text { both } x y x=x \text { and } y x y=y \\
\mathcal{L}: & x \mathcal{L} y & \text { iff } & \text { both } x y=x \text { and } y x=y \\
\mathcal{R}: & x \mathcal{R} y & \text { iff } & \text { both } x y=y \text { and } y x=x .
\end{array}
$$

For bands, $\mathcal{L}$ and $\mathcal{R}$ commute under the usual composition of relations, with the common outcome being $\mathcal{D}$, i.e., $\mathcal{L} \circ \mathcal{R}=\mathcal{R} \circ \mathcal{L}=\mathcal{R} \vee \mathcal{L}=\mathcal{D}$. Here $\mathcal{R} \vee \mathcal{L}$ denotes the join of the two relations. Moreover, we have the following fundamental result of Clifford and McLean [3, 10]:

Theorem 2.1. Given a band $(S ; \cdot)$, the relation $\mathcal{D}$ is a congruence for which $S / \mathcal{D}$ is the maximal semilattice image and each $\mathcal{D}$-class of $S$ is a maximal rectangular subalgebra of $S$. In brief, every band is a semilattice of rectangular bands.

So what do rectangular bands look like? First there are two basic cases. A left-zero band is a band $(L ; \cdot)$ with the trivial composition: $x y=x$. A right-zero band is a band $(R ; \cdot)$ with the trivial composition: $x y=y$. In other words, we either have just a single 
$\mathcal{L}$-class or just a single $\mathcal{R}$-class. Finally, there are products of both types, $L \times R$, and up to isomorphism, that is it. Thus a rectangular band may be pictured as a rectangular grid consisting of rows that are $\mathcal{R}$-classes and columns that are $\mathcal{L}$-classes.

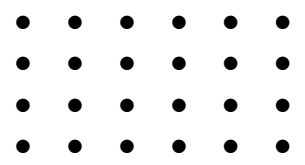

The product $x y$ of elements $x$ and $y$ is the unique element in the row of $x$ and the column of $y$. Given a rectangular band $(S ; \cdot)$ and $x$ in $S$, if $L$ denotes the $\mathcal{L}$-class of $x$ and $R$ denotes the $\mathcal{R}$-class of $S$, and $\varphi: L \times R \rightarrow S$ is defined by $\varphi(u, v)=u v \in S$, then $\varphi$ is an isomorphism of rectangular bands. Rectangular bands are precisely the bands that are anti-commutative in that $x y=y x$ iff $x=y$.

While bands have a very simple local structure - their rectangular $\mathcal{D}$-classes - it is not immediately clear how elements from different $\mathcal{D}$-classes combine under the binary operation.

A band is regular if the relations $\mathcal{L}$ and $\mathcal{R}$ are both congruences. Semilattices and rectangular bands are both regular. In the semilattice case $\mathcal{L}$ and $\mathcal{R}$ reduce to the identity relation, so that regularity is trivial. One might expect all bands to be regular, but that is not so. In the rectangular case there is more: $\mathcal{L}$ and $\mathcal{R}$ commute under composition, not only with each other, but with every congruence $\theta$ :

$$
\mathcal{L} \circ \theta=\theta \circ \mathcal{L}=\theta \vee \mathcal{L} \text { and } \mathcal{R} \circ \theta=\theta \circ \mathcal{R}=\theta \vee \mathcal{R}
$$

A double band is an algebra $(S ; \vee, \wedge)$ for which both reducts $(S ; \vee)$ and $(S ; \wedge)$ are bands. A lattice is thus a double band where both $(S ; \vee)$ and $(S ; \wedge)$ are semilattices that jointly satisfy the standard absorption identities for a lattice: $x \wedge(x \vee y)=x=x \vee(x \wedge y)$. A very general class of noncommutative lattices is as follows. A quasilattice is a double band that satisfies the following (modified) absorption identities:

$$
x \wedge(y \vee x \vee y) \wedge x=x=x \vee(y \wedge x \wedge y) \vee x .
$$

Note that if commutativity is assumed, both identities reduce to the absorption identities for a lattice.

A skew lattice is a noncommutative lattice that satisfies the dual absorption identities:

$$
\begin{aligned}
& x \wedge(x \vee y)=x=(x \vee y) \wedge x, \\
& x \vee(x \wedge y)=x=(x \wedge y) \vee x .
\end{aligned}
$$

A skew lattice is a quasilattice, but not conversely. In a quasilattice, both operations share common $\mathcal{D}$-classes that also form subalgebras, although on these classes both operations need not agree! Clearly, for a quasilattice $(S ; \vee, \wedge), \mathcal{D}$ is a congruence. Indeed, $S / \mathcal{D}$ is the maximal lattice image of $S$. This leads us to:

Definition 2.2. An antilattice is a double band $(S ; \vee, \wedge)$ for which both reducts, $(S ; \vee)$ and $(S ; \wedge)$, are rectangular bands, i.e., satisfy the identity $x y z=x z$ or equivalently $x y x=x$.

An antilattice is trivially a quasilattice. Conversely, each $\mathcal{D}$-class of a quasilattice is a subalgebra that is an antilattice. 
If the antilattice is a skew lattice, it is also called a rectangular skew lattice. As an antilattice, it is characterized by $x \wedge y=y \vee x$. D -classes of skew lattices are always rectangular skew lattices.

Similar to bands, a version of the Clifford-McLean Theorem holds:

Theorem 2.3. Given a quasilattice $(S ; \vee, \wedge)$, the relation $\mathcal{D}$ is the same for both, $(S ; \vee)$ and $(S ; \wedge)$. For this common congruence, the quotient algebra $S / \mathcal{D}$ is the maximal lattice image and each $\mathcal{D}$-class of $S$ is a maximal sub-antilattice of $S$. In brief, every quasilattice is a lattice of antilattices. (Compare Corollary 3 of [7]; see also [9].)

Antilattices have been studied, not only due to their connection to quasilattices, but also in connection with magic squares and finite planes. (See [8].)

Like quasilattices and semigroups, by definition antilattices do not have prescribed constants, thus making the empty set a viable subalgebra. In so doing, this allows for the existence of a complete lattice of subalgebras for any given antilattice.

\section{Regular antilattices}

Given an antilattice $(S ; \vee, \wedge)$, both reducts $(S ; \vee)$ and $(S ; \wedge)$ are regular in that $\mathcal{L}_{(\vee)}$ and $\mathcal{R}_{(\vee)}$ are congruences on $(S ; \vee)$, and likewise $\mathcal{L}_{(\wedge)}$ and $\mathcal{R}_{(\wedge)}$ are congruences on $(S ; \wedge)$. The antilattice is regular if all four equivalences are congruences for the whole algebra. In general, a quasilattice $(S ; \vee, \wedge)$ is regular if $\mathcal{L}_{(\vee)}, \mathcal{R}_{(\vee)}, \mathcal{L}_{(\wedge)}$ and $\mathcal{R}_{(\wedge)}$ are congruences of $(S ; \vee, \wedge)$. Skew lattices are regular, but in general, quasilattices need not be regular.

Theorem 3.1. Regular antilattices form a subvariety of the variety of antilattices.

Proof. We show that antilattices for which $\mathcal{L}_{(\vee)}$ is a congruence form a subvariety. To begin, in an antilattice $S, x \mathcal{L}_{(\vee)} u \vee x$ holds for all $u, x \in S$, and conversely, if $x \mathcal{L}_{(\vee)} x^{\prime}$, then trivially, $x^{\prime}=x^{\prime} \vee x$. Since $\mathcal{L}_{(\vee)}$ is already a congruence on the reduct $(S ; \vee)$, for $\mathcal{L}_{(\vee)}$ to be a congruence on $(S ; \wedge)$, precisely the following identities need to hold:

$$
(y \wedge x) \vee[y \wedge(u \vee x)]=y \wedge x \quad \& \quad[y \wedge(u \vee x)] \vee(y \wedge x)=y \wedge(u \vee x)
$$

and

$$
(x \wedge y) \vee[(u \vee x) \wedge y]=x \wedge y \quad \& \quad[(u \vee x) \wedge y] \vee(y \wedge x)=(u \vee x) \wedge y
$$

Thus this class of antilattices indeed forms a subvariety. Similar remarks verify the same claim for $\mathcal{R}_{(\vee)}, \mathcal{L}_{(\wedge)}$ and $\mathcal{R}_{(\wedge)}$. The theorem now follows.

Since $\mathcal{L}$ and $\mathcal{R}$ commute under composition with all congruences on a rectangular band, $\mathcal{L}_{(\vee)} \circ \mathcal{L}_{(\wedge)}=\mathcal{L}_{(\wedge)} \circ \mathcal{L}_{(\vee)} ; \mathcal{L}_{(\vee)} \circ \mathcal{R}_{(\wedge)}=\mathcal{R}_{(\wedge)} \circ \mathcal{L}_{(\vee)} ; \mathcal{R}_{(\vee)} \circ \mathcal{L}_{(\wedge)}=\mathcal{L}_{(\wedge)} \circ \mathcal{R}_{(\vee)} ;$ and $\mathcal{R}_{(\vee)} \circ \mathcal{R}_{(\wedge)}=\mathcal{R}_{(\wedge)} \circ \mathcal{R}_{(\vee)}$ hold for regular antilattices. All four outcomes are thus congruences on the antilattice, and indeed form the join congruences of the respective pairs of congruences.

An antilattice $(S ; \vee, \wedge)$ is flat if the reduct $(S ; \vee)$ is either a left-0 semigroup $(x \vee y=$ $x$ ) or a right- 0 semigroup $(x \vee y=y)$, and likewise the reduct $(S ; \wedge)$ is either a left-0 semigroup $(x \wedge y=x)$ or a right-0 semigroup $(x \wedge y=y)$. That is, for each operation, either $\mathcal{D}=\mathcal{L}$ or $\mathcal{D}=\mathcal{R}$. Clearly there are 4 distinct classes of flat antilattices:

- the class $\mathbf{A}_{\mathcal{L} \mathcal{L}}$ of all antilattices where $x \vee y=x=x \wedge y$. 
- the class $\mathbf{A}_{\mathcal{L} \mathcal{R}}$ of all antilattices where $x \vee y=x$ but $x \wedge y=y$.

- the class $\mathbf{A}_{\mathcal{R} \mathcal{L}}$ of all antilattices where $x \vee y=y$ but $x \wedge y=x$.

- the class $\mathbf{A}_{\mathcal{R} \mathcal{R}}$ of all antilattices where $x \vee y=y=x \wedge y$.

Clearly each class is a subvariety of regular antilattices. What is more:

Lemma 3.2. Flat antilattices $S$ and $T$ of the same class are isomorphic if and only if they have the same cardinality. When the latter is the case, an isomorphism is given by any bijection between $S$ and $T$.

Theorem 3.3 (Decomposition Theorem). Every nonempty regular antilattice $(S ; \vee, \wedge)$ factors into the direct product $S_{\mathcal{L L}} \times S_{\mathcal{L R}} \times S_{\mathcal{R} \mathcal{L}} \times S_{\mathcal{R} \mathcal{R}}$ of its four maximal flat images, one from each class above, with the respective factors being unique up to isomorphism.

Proof. The factorization is obtained by first factoring with respect to say $\vee$ :

$$
S \cong S / \mathcal{R}_{(\vee)} \times S / \mathcal{L}_{(\vee)}
$$

to get two factors for which the $\vee$-operation is flat. Then similarly factor both factors further with respect to the relevant $\mathcal{R}_{(\wedge)}$ and $\mathcal{L}_{(\wedge)}$ congruences to get four flat factors:

$$
S \cong S_{\mathcal{L L}} \times S_{\mathcal{L R}} \times S_{\mathcal{R} \mathcal{L}} \times S_{\mathcal{R} \mathcal{R}}
$$

where: $S_{\mathcal{L L}}=S /\left(\mathcal{R}_{(\vee)} \vee \mathcal{R}_{(\wedge)}\right) ; S_{\mathcal{L} \mathcal{R}}=S /\left(\mathcal{R}_{(\vee)} \vee \mathcal{L}_{(\wedge)}\right) ; S_{\mathcal{R} \mathcal{L}}=S /\left(\mathcal{L}_{(\vee)} \vee \mathcal{R}_{(\wedge)}\right)$ and $S_{\mathcal{R} \mathcal{R}}=S /\left(\mathcal{L}_{(\vee)} \vee \mathcal{L}_{(\wedge)}\right)$

Further factorization can take place. But first, given a positive integer $n$, let $\mathbf{n}_{\mathcal{L} \mathcal{L}}, \mathbf{n}_{\mathcal{L} \mathcal{R}}$, $\mathbf{n}_{\mathcal{R} \mathcal{L}}$ and $\mathbf{n}_{\mathcal{R} \mathcal{R}}$ denote the relevant flat antilattices on the set $\{1,2,3, \ldots, n\}$. This leads us to the following finite version of the Decomposition Theorem:

Theorem 3.4. Let $(S ; \vee, \wedge)$ be a nonempty finite regular antilattice with the above factorization $S_{\mathcal{L L}} \times S_{\mathcal{L R}} \times S_{\mathcal{R} \mathcal{L}} \times S_{\mathcal{R} \mathcal{R}}$. If $n_{\mathcal{L L}}=\left|S_{\mathcal{L} \mathcal{L}}\right|, n_{\mathcal{L} \mathcal{R}}=\left|S_{\mathcal{L R}}\right|$, etc., then

$$
S \cong \mathbf{n}_{\mathcal{L L}} \times \mathbf{n}_{\mathcal{L R}} \times \mathbf{n}_{\mathcal{R} \mathcal{L}} \times \mathbf{n}_{\mathcal{R} \mathcal{R}}
$$

Clearly these four parameters characterize $(S ; \vee, \wedge)$. It is also clear that factorization can continue on each of the four factors. For instance say $\left|S_{\mathcal{L} \mathcal{L}}\right|=180=4 \times 5 \times 9$. Then we have $S_{\mathcal{L} \mathcal{L}} \cong\left(\mathbf{2}_{\mathcal{L L}}\right)^{2} \times\left(\mathbf{3}_{\mathcal{L L}}\right)^{2} \times \mathbf{5}_{\mathcal{L} \mathcal{L}}$. Up to isomorphism, the only 1-point algebra is the trivial algebra $\mathbf{1}=\{0\}$.

Corollary 3.5. A regular antilattice $(S ; \vee, \wedge)$ is directly irreducible iff $|S|$ is either 1 or a prime. Every finite regular antilattice of order $>1$ thus factors into a direct product of finitely many flat antilattices of prime order.

Corollary 3.6. A regular antilattice $(S ; \vee, \wedge)$ is subdirectly irreducible iff either $|S|=1$ or $|S|=2$. Every (finite) regular antilattice of order $>1$ is thus isomorphic to a subdirect product of (finitely) many flat antilattices of order 2.

A sub-(pseudo)variety of regular antilattices is positive, if it is not the sub-variety $\{\emptyset\}$.

Corollary 3.7. The lattice of all positive subvarieties of regular antilattices is a Boolean algebra with 16 elements and 4 atoms: $\mathbf{A}_{\mathcal{L L}}, \mathbf{A}_{\mathcal{L} \mathcal{R}}, \mathbf{A}_{\mathcal{R} \mathcal{L}}$ and $\mathbf{A}_{\mathcal{R} \mathcal{R}}$ (see Figure 1). 
Corollary 3.8. The lattice of all positive sub-pseudovarieties of finite regular antilattices is a Boolean algebra with 16 elements and the four atoms as above, but with their respective classes now restricted to finite algebras: ${ }_{f} \mathbf{A}_{\mathcal{L L},{ }_{f}} \mathbf{A}_{\mathcal{L} \mathcal{R},{ }_{f}} \mathbf{A}_{\mathcal{R} \mathcal{L}}$ and ${ }_{f} \mathbf{A}_{\mathcal{R} \mathcal{R}}$.

We will take a closer look at the positive subvarieties involved in the fourth section.

What can one say about the congruence lattice of a regular antilattice? To begin observe that the four classes of flat antilattces are mutually term equivalent with each other and with the class of all left-zero semigroups and also the class of all right-zero semigroups. In all these special cases the congruence lattice is precisely the full lattice $\Pi(S)$ of all equivalences of the underlying set $S$. Following the situation for rectangular bands in general, we have:

Theorem 3.9. Let a nonempty regular antilattice $(S ; \vee, \wedge)$ be factored into the direct product of its four maximal flat images: $S_{\mathcal{L} \mathcal{L}} \times S_{\mathcal{L R}} \times S_{\mathcal{R} \mathcal{L}} \times S_{\mathcal{R} \mathcal{R}}$. Then the congruence lattice of $S$ is given by $\Pi\left(S_{\mathcal{L} \mathcal{L}}\right) \times \Pi\left(S_{\mathcal{L} \mathcal{R}}\right) \times \Pi\left(S_{\mathcal{R} \mathcal{L}}\right) \times \Pi\left(S_{\mathcal{R} \mathcal{R}}\right)$. That is, if the elements if $S$ are expressed as 4-tuples $(x, y, z, w)$ given by the factorization, then each congruence $\theta$ on $S$ can be represented as a 4 -tuple $\left(\theta_{\mathcal{L L}}, \theta_{\mathcal{L R}}, \theta_{\mathcal{R} \mathcal{L}}, \theta_{\mathcal{R} \mathcal{R}}\right)$ of congruences on each factor in that:

$$
(x, y, z, w) \theta\left(x^{\prime}, y^{\prime}, z^{\prime}, w^{\prime}\right) \quad \text { iff } \quad x \theta_{\mathcal{L L}} x^{\prime}, y \theta_{\mathcal{L} \mathcal{R}} y^{\prime}, z \theta_{\mathcal{R} \mathcal{L}} z^{\prime} \& w \theta_{\mathcal{R} \mathcal{R}} w^{\prime}
$$

Conversely, in this manner every such 4-tuple of congruences defines a congruence on the full antilattice $S$.

In similar fashion:

Theorem 3.10. Given a nonempty regular antilattice $S$ with factorization $S_{\mathcal{L L}} \times S_{\mathcal{L R}} \times$ $S_{\mathcal{R} \mathcal{L}} \times S_{\mathcal{R} \mathcal{R}}$, if $a=\left|S_{\mathcal{L} \mathcal{L}}\right|, b=\left|S_{\mathcal{L R}}\right|, c=\left|S_{\mathcal{R} \mathcal{L}}\right|$ and $d=\left|S_{\mathcal{R} \mathcal{R}}\right|$, then the number of subalgebras of $S$ is:

$$
1+\left(2^{a}-1\right)\left(2^{b}-1\right)\left(2^{c}-1\right)\left(2^{d}-1\right) .
$$

One can ask: given a positive integer $n \geq 1$, up to isomorphism, how many nonisomorphic regular antilattices are there of size n? By Theorem 3.4 it is the number $\rho(n)$ of 4 -fold positive factorizations $a b c d$ of $n$, where the order of the factors $a, b, c, d$ is important. Here $a$ is the size of the $\mathcal{L} \mathcal{L}$-factor, $b$ is the size of the $\mathcal{L} \mathcal{R}$-factor, etc.

To begin, thanks to Corollary 3.5 , given the prime power factorization $n=2^{e_{2}} 3^{e_{3}}$ $5^{e_{5}} \cdots p_{k}^{e_{p_{k}}}$ :

$$
\rho(n)=\rho\left(2^{e_{2}}\right) \rho\left(3^{e_{3}}\right) \rho\left(5^{e_{5}}\right) \cdots \rho\left(p_{k}^{e_{p_{k}}}\right) .
$$

Thus things can be reduced to calculating $\rho\left(p^{e}\right)$ for any prime power $p^{e}$.

From a combinatorial perspective, this is equivalent to asking in how many distinct ways can $e$ identical balls be distributed into 4 labeled boxes. This question has a simple answer:

$$
\rho\left(p^{e}\right)=\left(\begin{array}{c}
e+3 \\
3
\end{array}\right)
$$

By putting all these together we obtain the following closed formula for $\rho(n)$ :

Theorem 3.11. Let $n$ have the following prime power factorization $n=2^{e_{2}} 3^{e_{3}} 5^{e_{5}} \cdots p_{k}^{e_{p_{k}}}$ : Then

$$
\rho(n)=\left(\begin{array}{c}
e_{2}+3 \\
3
\end{array}\right)\left(\begin{array}{c}
e_{3}+3 \\
3
\end{array}\right)\left(\begin{array}{c}
e_{5}+3 \\
3
\end{array}\right) \cdots\left(\begin{array}{c}
e_{p_{k}}+3 \\
3
\end{array}\right) .
$$




\section{See also Table 1.}

One can ask a more general question: In how many distinct ways can $e$ identical balls be distributed into $k$ labeled boxes? This question has analogous answer, namely $\left(\begin{array}{c}e+k-1 \\ k-1\end{array}\right)$; see, for instance [1]. We will use some of these in the next section. Note that such an ordered partition of an integer $n$ into $k$ possibly empty parts is sometimes called a composition; see [1].

\section{Semi-flat antilattices and other subvarieties}

An antilattice $(S ; \vee, \wedge)$ is semi-flat if either $(S ; \vee)$ or $(S ; \wedge)$ is flat. Flat antilattices are trivially semi-flat. The class of all semi-flat antilattices consists of four distinct subclasses that are not necessarily disjoint:

- $\mathbf{A}_{\mathcal{L} \#}$, the class of all antilattices $(S ; \vee, \wedge)$ s.t. $(S ; \vee)$ is a left-0 band;

- $\mathbf{A}_{\mathcal{R} \#}$, the class of all antilattices $(S ; \vee, \wedge)$ s.t. $(S ; \vee)$ is a right-0 band;

- $\mathbf{A}_{\# \mathcal{L}}$, the class of all antilattices $(S ; \vee, \wedge)$ s.t. $(S ; \wedge)$ is a left-0 band;

- $\mathbf{A}_{\# \mathcal{R}}$, the class of all antilattices $(S ; \vee, \wedge)$ s.t. $(S ; \wedge)$ is a right- 0 band.

Theorem 4.1. These four classes are subvarieties of the variety of regular antilattices.

Proof. First observe that each is at least a subvariety in the variety of all antilattices. We show this for $\mathbf{A}_{\mathcal{L} \#}$, the other cases being similar. The identity characterizing $\mathbf{A}_{\mathcal{L} \#}$ in the variety of antilattices is clearly $x \vee y=x$. Thus $\mathbf{A}_{\mathcal{L} \#}$ is indeed a subvariety of antilattices. To see that all semi-flat antilattices are regular, again we need only consider, say, $\mathbf{A}_{\mathcal{L} \# \text {. So }}$ let $(S ; \vee, \wedge)$ be an antilattice for which $(S ; \vee)$ is a left zero-band. Thus $\mathcal{L}_{(\vee)}$ is the universal equivalence $\nabla$ on $S$, and thus trivially a congruence on $(S ; \wedge)$ while $\mathcal{R}_{(\vee)}$ is the identity equivalence and thus again trivially a congruence on $(S ; \wedge)$. Next consider $\mathcal{L}_{(\wedge)}$ and $\mathcal{R}_{(\wedge)}$. Being congruences on $(S ; \wedge)$, they are at least equivalences on $S$. But all equivalences on $S$ are congruences on the left zero-band $(S ; \vee)$, and thus $\mathcal{L}_{(\wedge)}$ and $\mathcal{R}_{(\wedge)}$ are congruences on $(S ; \vee, \wedge)$.

Consider next the following diagram.

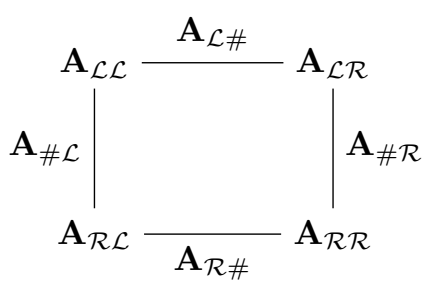

The four flat varieties occupy the middle rectangle. If two distinct flat varieties are adjacent on this rectangle, their join variety is the semi-flat variety labeling the line between them. But if they are diagonal opposites, we have the following:

- $\mathbf{A}_{\mathcal{L} \mathcal{L}} \vee \mathbf{A}_{\mathcal{R} \mathcal{R}}=$ the subvariety of antilattices for which $x \vee y=x \wedge y$.

- $\mathbf{A}_{\mathcal{L} \mathcal{R}} \vee \mathbf{A}_{\mathcal{R} \mathcal{L}}=$ the subvariety of antilattices for which $x \vee y=y \wedge x$. 
These are the antilattice subvarieties that are, respectively, skew* lattices or skew lattices.

Next are the four double joins. Consider $\mathbf{A}_{\mathcal{L} \mathcal{L}} \vee \mathbf{A}_{\mathcal{L R}} \vee \mathbf{A}_{\mathcal{R} \mathcal{L}}$. It consists of regular antilattices for which the $\mathbf{A}_{\mathcal{R} \mathcal{R}}$-factor is trivial. Since $\vee$ and $\wedge$ are idempotent, this reduces to no nontrivial $\mathbf{A}_{\mathcal{R} \mathcal{R}}$-subalgebra occurring in the given antilattice. More briefly, no copy of $\mathbf{2}_{\mathcal{R} \mathcal{R}}$ occurs as a subalgebra. This is guaranteed by the identity $x \vee(x \wedge y)=x$ (that is equivalent to the implication: $\left.u \mathcal{R}_{(\wedge)} v \Rightarrow u \mathcal{L}_{(\vee)} v\right)$ along with its $\vee-\wedge$ dual. This subvariety is, of course, the Boolean complement $\mathbf{A}_{\mathcal{R} \mathcal{R}}^{C}$ of $\mathbf{A}_{\mathcal{R} \mathcal{R}}$. The three other double joins are treated similarly to obtain:

$$
\begin{aligned}
& \mathbf{A}_{\mathcal{L} \mathcal{L}} \vee \mathbf{A}_{\mathcal{L} \mathcal{R}} \vee \mathbf{A}_{\mathcal{R} \mathcal{R}}=\mathbf{A}_{\mathcal{R} \mathcal{L}}^{C}, \\
& \mathbf{A}_{\mathcal{L L}} \vee \mathbf{A}_{\mathcal{R} \mathcal{L}} \vee \mathbf{A}_{\mathcal{R} \mathcal{R}}=\mathbf{A}_{\mathcal{L} \mathcal{R}}^{C}, \\
& \mathbf{A}_{\mathcal{L} \mathcal{R}} \vee \mathbf{A}_{\mathcal{R} \mathcal{L}} \vee \mathbf{A}_{\mathcal{R} \mathcal{R}}=\mathbf{A}_{\mathcal{L} \mathcal{L}}^{C} .
\end{aligned}
$$

Finally, above these four lies the full variety of all regular antilattices and just below the four flat cases lies the variety of trivial 1-point algebras. The resulting lattice of all subvarieties of regular antilattices is, of course, isomorphic to the lattice of all subsets of any 4-element set, which brings us back to Corollary 3.7.

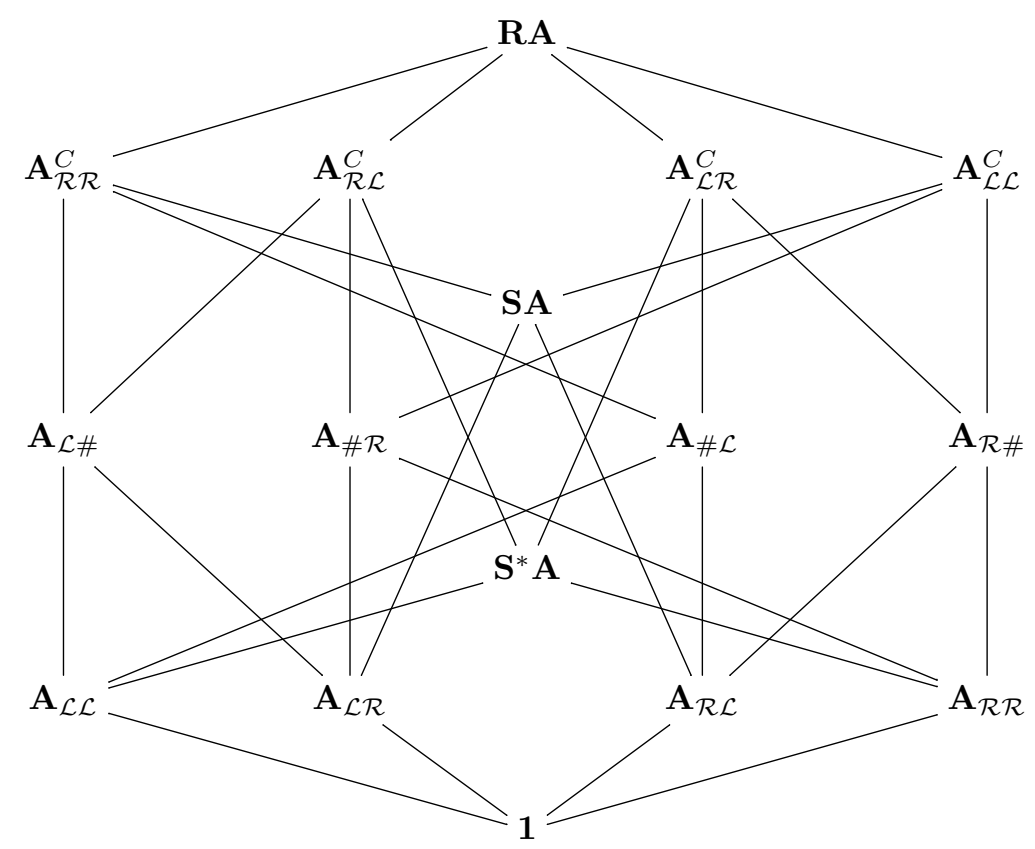

Figure 1: The Hasse diagram of the Boolean lattice of all positive subvarieties of antilattices.

The Hasse diagram of this Boolean lattice is explained in Table 1.

Equipped with all necessary tools we may now perform enumeration of the pseudovariety of finite regular antilattices and their sub-pseudo-varieties; see Table 2. These sequences can be found in OEIS [11]. 
Table 1: The 16 positive subvarieties of regular antilattices.

\begin{tabular}{l|l|c} 
Symbol & Subvariety & $\rho\left(p^{e}\right)$ \\
\hline \hline $\mathbf{R} \mathbf{A}$ & regular antilattices & $\left(\begin{array}{c}e+3 \\
3\end{array}\right)$ \\
\hline $\mathbf{A}_{\mathcal{R} \mathcal{R}}^{C}$ & complement of $\mathbf{A}_{\mathcal{R} \mathcal{R}}$ & $\left(\begin{array}{c}e+2 \\
2\end{array}\right)$ \\
$\mathbf{A}_{\mathcal{R} \mathcal{L}}^{C}$ & complement of $\mathbf{A}_{\mathcal{R} \mathcal{L}}$ & $\left(\begin{array}{c}e+2 \\
2\end{array}\right)$ \\
$\mathbf{A}_{\mathcal{L} \mathcal{R}}^{C}$ & complement of $\mathbf{A}_{\mathcal{L} \mathcal{R}}$ & $\left(\begin{array}{c}e+2 \\
2\end{array}\right)$ \\
$\mathbf{A}_{\mathcal{L} \mathcal{L}}^{C}$ & complement of $\mathbf{A}_{\mathcal{L} \mathcal{L}}$ & $\left(\begin{array}{c}e+2 \\
2\end{array}\right)$ \\
\hline $\mathbf{S} \mathbf{A}$ & skew antilattices & $\left(\begin{array}{c}e+1 \\
1\end{array}\right)=e+1$ \\
$\mathbf{A}_{\mathcal{L} \#}$ & $\mathcal{L}^{*}$ semi-flat & $\left(\begin{array}{c}e+1 \\
1\end{array}\right)=e+1$ \\
$\mathbf{A}_{\# \mathcal{R}}$ & ${ }^{*} \mathcal{R}$ semi-flat & $\left(\begin{array}{c}e+1 \\
1\end{array}\right)=e+1$ \\
$\mathbf{A}_{\# \mathcal{L}}$ & ${ }^{*} \mathcal{L}$ semi-flat & $\left(\begin{array}{c}e+1 \\
1\end{array}\right)=e+1$ \\
$\mathbf{A}_{\mathcal{R} \#}$ & $\mathcal{R}^{*}$ semi-flat & $\left(\begin{array}{c}e+1 \\
1\end{array}\right)=e+1$ \\
$\mathbf{S}^{*} \mathbf{A}$ & skew antilattices & $\left(\begin{array}{c}e+1 \\
1\end{array}\right)=e+1$ \\
\hline $\mathbf{A}_{\mathcal{L L}}$ & $\mathcal{L} \mathcal{L}$-flat & $\left(\begin{array}{c}e+0 \\
0\end{array}\right)=1$ \\
$\mathbf{A}_{\mathcal{L R}}$ & $\mathcal{L} \mathcal{R}$-flat & $\left(\begin{array}{c}e+0 \\
0\end{array}\right)=1$ \\
$\mathbf{A}_{\mathcal{R} \mathcal{L}}$ & $\mathcal{R} \mathcal{L}$-flat & $\left(\begin{array}{c}e+0 \\
0\end{array}\right)=1$ \\
$\mathbf{A}_{\mathcal{R} \mathcal{R}}$ & $\mathcal{R} \mathcal{R}$-flat & $\left(\begin{array}{c}e+0 \\
0\end{array}\right)=1$ \\
\hline $\mathbf{1}$ & trivial antilattice & 1 if $e=0$ \\
& &
\end{tabular}

Table 2: Enumeration of small regular antilattices and their subvarieties.

\begin{tabular}{|c|c|c|c|c|c|}
\hline$n$ & RA & $\begin{array}{c}\mathbf{A}_{\mathcal{R} \mathcal{R}}^{C}, \mathbf{A}_{\mathcal{R} \mathcal{L}}^{C}, \\
\mathbf{A}_{\mathcal{L} \mathcal{R}}^{C}, \mathbf{A}_{\mathcal{L} \mathcal{L}}^{C}\end{array}$ & $\begin{array}{l}\mathbf{S A}, \mathbf{A}_{\mathcal{L} \#}, \mathbf{A}_{\# \mathcal{R}}, \\
\mathbf{A}_{\# \mathcal{L}}, \mathbf{A}_{\mathcal{R} \#}, \mathbf{S}^{*} \mathbf{A}\end{array}$ & $\begin{array}{l}\mathbf{A}_{\mathcal{L} \mathcal{L}}, \mathbf{A}_{\mathcal{L R}}, \\
\mathbf{A}_{\mathcal{R} \mathcal{L}}, \mathbf{A}_{\mathcal{R} \mathcal{R}}\end{array}$ & 1 \\
\hline$\overline{\text { OEIS }}$ & A007426 & A007425 & A000005 & A000012 & \\
\hline 1 & 1 & 1 & 1 & 1 & 1 \\
\hline 2 & 4 & 3 & 2 & 1 & 0 \\
\hline 3 & 4 & 3 & 2 & 1 & 0 \\
\hline 4 & 10 & 6 & 3 & 1 & 0 \\
\hline 5 & 4 & 3 & 2 & 1 & 0 \\
\hline 6 & 16 & 9 & 4 & 1 & 0 \\
\hline 7 & 4 & 3 & 2 & 1 & 0 \\
\hline 8 & 20 & 10 & 4 & 1 & 0 \\
\hline 9 & 10 & 6 & 3 & 1 & 0 \\
\hline 10 & 16 & 9 & 4 & 1 & 0 \\
\hline 11 & 4 & 3 & 2 & 1 & 0 \\
\hline 12 & 40 & 18 & 6 & 1 & 0 \\
\hline 13 & 4 & 3 & 2 & 1 & 0 \\
\hline 14 & 16 & 9 & 4 & 1 & 0 \\
\hline 15 & 16 & 9 & 4 & 1 & 0 \\
\hline 16 & 35 & 15 & 5 & 1 & 0 \\
\hline
\end{tabular}




\section{References}

[1] M. Bóna, A Walk Through Combinatorics: An Introduction to Enumeration and Graph Theory, World Scientific, Hackensack, New Jersey, 2nd edition, 2006, doi:10.1142/6177.

[2] S. Burris and H. P. Sankappanavar, A Course in Universal Algebra, volume 78 of Graduate Texts in Mathematics, Springer-Verlag, New York, 1981, http://www.math. uwaterloo.ca/ snburris/htdocs/ualg.html.

[3] A. H. Clifford, Semigroups admitting relative inverses, Ann. of Math. 42 (1941), 1037-1049, doi: $10.2307 / 1968781$.

[4] A. H. Clifford and G. B. Preston, The Algebraic Theory of Semigroups, Volume I, volume 7 of Mathematical Surveys, American Mathematical Society, Providence, Rhode Island, 1961, doi:10.1090/surv/007.1.

[5] P.-A. Grillet, Semigroups: An Introduction to the Structure Theory, volume 193 of Monographs and Textbooks in Pure and Applied Mathematics, Marcel Dekker, New York, 1995.

[6] J. M. Howie, Fundamentals of Semigroup Theory, volume 12 of London Mathematical Society Monographs (New Series), The Clarendon Press, Oxford University Press, New York, 1995.

[7] G. Laslo and J. Leech, Green's equivalences on noncommutative lattices, Acta Sci. Math. (Szeged) 68 (2002), 501-533, http://pub.acta.hu/acta/ showCustomerArticle.action?id=2881\&dataObject Type=article.

[8] J. Leech, Magic squares, finite planes and simple quasilattices, Ars Combin. 77 (2005), 75-96.

[9] J. Leech, My journey into noncommutative lattices and their theory, Art Discrete Appl. Math. 2 (2019), \#P2.01, doi:10.26493/2590-9770.1282.e7a.

[10] D. McLean, Idempotent semigroups, Amer. Math. Monthly 61 (1954), 110-113, doi:10.2307/ 2307797.

[11] N. J. A. Sloane (ed.), The On-Line Encyclopedia of Integer Sequences, published electronically at https://oeis.org. 